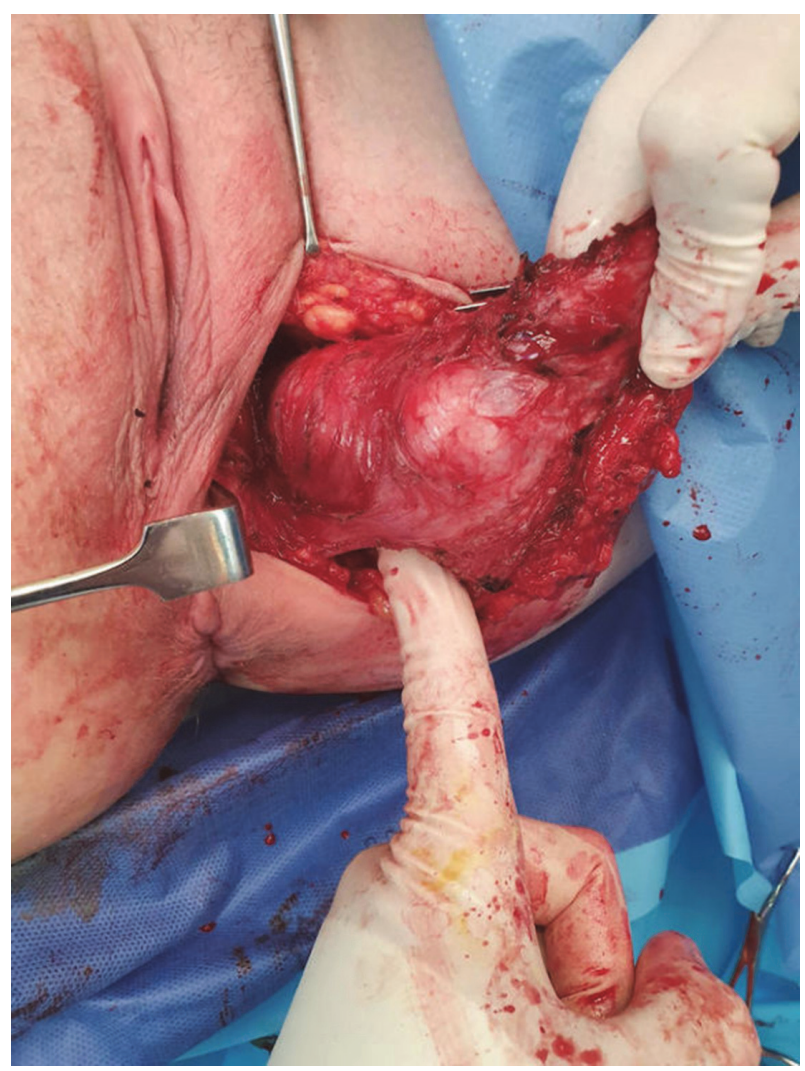

Abstract EPV260/\#451 Figure 2

preoperative imaging, this approach is safe and effective to achieve complete surgical resection, avoiding relapse. Other risk factors include incontinence and sensory defecit. The procedure requires an in depth knowledge of the pelvic anatomical spaces, their contents and boundaries.

\section{EPV261/\#46 TOTAL LAPAROSCOPIC HYSTERECTOMY IN MORBIDLY OBESE PATIENTS - REDUCING THE RISK}

E Van Der Zanden, F Testa, C White, S Kaushik, P Larsen-Disney, F Drews, S Baron* Brighton and Sussex University Hospital NHS Trust, Gynaecological Oncology, Brighton, UK

\subsection{6/ijgc-2021-IGCS.332}

Objectives To evaluate whether a consultant 'buddy' operating approach improves on intra-operative and post-operative outcomes in patients undergoing total laparoscopic hysterectomy (TLH) for endometrial cancer who are extremely and morbidly obese.

Methods A prospectively selected cohort of 25 patients with a BMI 47-70 undergoing TLH was divided into two groups according to whether the first assistant to the Gynae-Oncology consultant was a registrar, or a consultant ('buddy operating'). Anaesthetic time, operating time, intraoperative estimated blood loss (EBL), requirement for high dependency unit (HDU) bed and length of stay (LOS) were compared.

Results Average 'buddy' operating time was significantly shorter compared to the registrar-assistant group $(01: 31 \mathrm{~h}$ vs $01: 59 \mathrm{~h}$ respectively; $\mathrm{p}<0.001)$; a similar trend was seen with the average total anaesthetic time $(02: 48 \mathrm{~h}$ vs $03: 23 \mathrm{~h}$ respectively; $\mathrm{p}<0.001)$. EBL was less in the 'buddy operating' group (39 mls) vs registrar-assistant group (169 mls; $\mathrm{p}<0.001)$. Post- operatively, LOS was shorter in the 'buddy operating' group as compared to the registrar-assistant, though not significantly so $(1.13$ vs 1.59 days; $p=0.109) .2$ of the total patients $(8 \%)$ required a one-night stay in HDU for observation due to their co-morbidities, both in the registrar-assistant group. Mean BMI, age, ASA and comorbidities were similar in the two groups.

Conclusions In patients with a significantly raised BMI, TLHs by two consultants vs consultant and registrar are associated with better intra and post-operative outcomes, including reduced overall anaesthetic time, operating time, and EBL. There is an association with a reduced length of overall hospital stay, though this was not significant.

\section{EPV262/\#462 FUNCTIONAL NEOVAGINA FORMATION USING VERTICAL RECTUS ABDOMINIS MUSCULOCUTANEOUS (VRAM) FLAP FOLLOWING PELVIC EXENTERATION}

${ }^{1} \mathrm{~S}$ Smyth*, ${ }^{1} \mathrm{C}$ Pappa, ${ }^{1} \mathrm{H}$ Jiang, ${ }^{2} \mathrm{R}$ Mykula, ${ }^{1} \mathrm{M}$ Alazzam. ${ }^{1}$ Oxford University Hospitals NHS Foundation Trust, Gynaecological Oncology, Oxford, UK; ${ }^{2}$ Oxford University Hospitals NHS Foundation Trust, Plastic Surgery, Oxford, UK

\subsection{6/ijgc-2021-IGCS.333}

Objectives Cervical cancer is common in younger women, with risks of surgery for advanced disease including altered body image and psychosexual dysfunction. This specialist technique highlights multidisciplinary management whilst minimising complications.

Methods A 42-year-old female with a stage 1B2 lymph node positive cervical adenocarcinoma managed with primary chemoradiotherapy underwent total pelvic infralevator exenteration for recurrence; with vulval sparing and VRAM reconstruction of the pelvic floor, perineum and neovagina formation.

Results Following preoperative marking and perforating vessel Doppler identification of the anterior abdominal wall; the anterior rectus sheath was preserved to the medial row and exenteration procedure completed with staggered urostomy

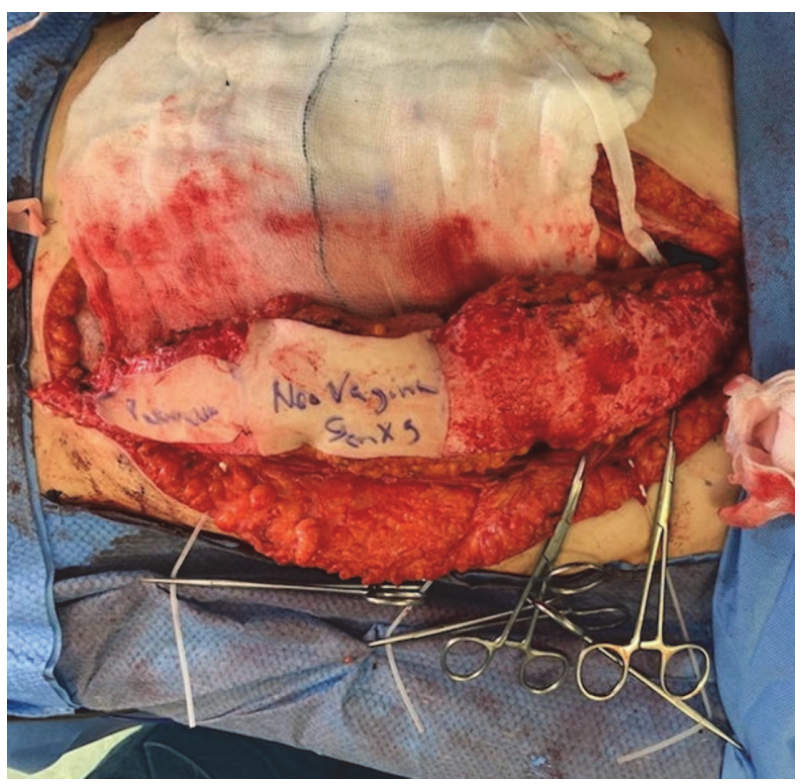

Abstract EPV262/\#462 Figure 1 


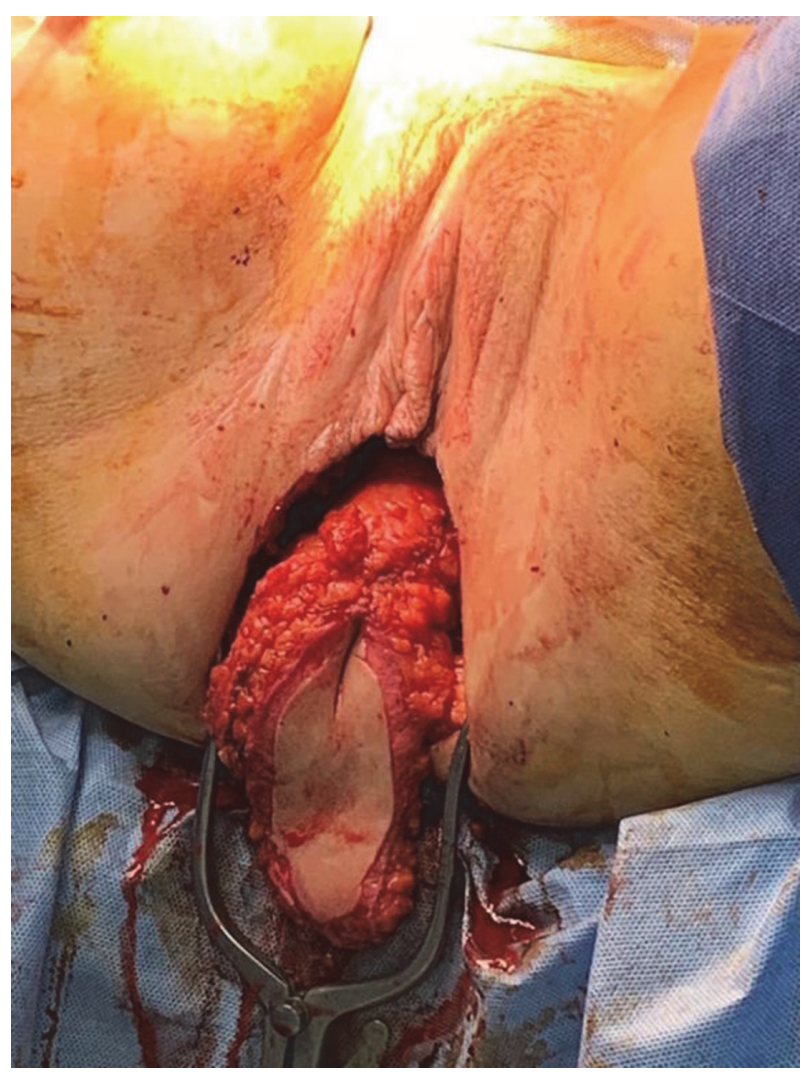

Abstract EPV262/\#462 Figure 2

and ileostomy. The VRAM (including skin, subcutaneous tissue, sheath and muscle) was then completed along the lateral aspect with preservation of the rectus abdominus insertion pedicle including inferior epigastric artery. The flap was rotated to the perineum with tubing of the neovagina and deepithelization for pelvic floor reconstruction. The abdomen was closed with a vicryl mesh insert.

Conclusions VRAM flap provides a reliable blood supply and viable tissue from a non-irradiated site with acceptable scarring. This reduces issues with empty pelvic syndrome and allows for ligation of the internal iliac artery. The patient made an uneventful recovery with retained sensation of the remaining vulva and flap. She continues under surveillance and vaginal mechanical dilation regime. We emphasise preservation of appearance and function in achieving patient satisfaction.

\section{EPV263/\#470 INITIAL EXPERIENCE WITH THE ENHANCED RECOVERY AFTER SURGERY (ERAS) PROTOCOL IN GYNECOLOGY AT A TERTIARY ACADEMIC MEDICAL CENTER}

J Noh*, C-H Choi, T-J Kim, J-W Lee, Y-Y Lee. Samsung Medical Center, Obstetrics and Gynecology, Seoul, Korea, Republic of

\subsection{6/ijgc-2021-IGCS.334}

Objectives The aim of this study was to describe the early experience with ERAS protocols for gynecologic surgeries at an urban academic tertiary medical center.

Methods The target population was the women who underwent various types of gynecologic surgeries for both benign and malignant diseases between October 2020 and January
2021. Two separate analyses were performed: a case-matched analysis between ERAS vs. non-ERAS cohorts and a retrospective cohort comparative analysis between ERAS vs. historical non-ERAS comparison groups.

Results A total of 200 patients were evaluated (122 patients in the ERAS group vs. 78 patients in the non-ERAS group). Fasting times were significantly shorter in the ERAS cohort group (26.3 vs. 40.1 hours for solid food, 6.9 vs. 15.4 hours for clear liquid in the ERAS group vs. non-ERAS group, both $\mathrm{p}$ values $<0.01)$. The use of opioid analgesia was also significantly lower (0.3 vs. 1.8 vials in the ERAS group vs. nonERAS group, p-value <0.01), whereas NSAIDs and acetaminophen use was more frequent in the ERAS group. The patients in the ERAS group reported less post-operative pain, feelings of hunger and thirst, and greater amount of exercise. The length of hospital stay did not differ between the two groups. These benefits of the ERAS protocols were more significant in the patients who underwent laparotomic surgeries than those who underwent laparoscopic surgeries. Similar patterns of the results were observed from the historical comparison analysis. Conclusions The ERAS protocols improved post-operative recovery after various gynecologic surgeries.

\section{EPV264/\#512 DOES NEGATIVE PRESSURE WOUND THERAPY REDUCE SURGICAL SITE INFECTION IN ENDOMETRIAL CANCER PATIENTS UNDERGOING LAPAROTOMY? A MULTICENTRE RETROSPECTIVE COHORT STUDY}

${ }^{1} \mathrm{~A}$ Montgomery*, ${ }^{2} \mathrm{~L}$ Li, ${ }^{3} \mathrm{M}$ Boo, ${ }^{4} \mathrm{M}$ Maul, ${ }^{2} \mathrm{~K}$ Da Silva, ${ }^{5} \mathrm{~A}$ Klasan, ${ }^{6} \mathrm{~L}$ Eva, ${ }^{6} \mathrm{C}$ Bergzoll. ${ }^{1}$ East Surrey Hospital, Oandg, Redhill, UK; ${ }^{2}$ Middlemore Hospital, Oandg, Auckland, New Zealand; ${ }^{3}$ Christchurch Hospital, Gynaecological Oncology, Christchurch, New Zealand; ${ }^{4}$ Northshore Hospital, Oandg, Auckland, New Zealand; ${ }^{5}$ Kepler University Hospital, Orthopaedics, Linz, Austria; ${ }^{6}$ National Women's Health, Gynaecologic Oncology, Auckland, New Zealand

\subsection{6/ijgc-2021-IGCS.335}

Objectives To establish the rate of surgical site infection in patients with endometrial carcinoma undergoing laparotomy using standard surgical dressings compared to those using negative wound pressure therapy (NPWT).

Methods Retrospective cohort analysis of 398 patients who underwent a laparotomy for endometrial carcinoma between 2013-2014 and 2018-2019 across three hospitals in New Zealand, to compare the effect of introduction of NPWT. SSI, wound dehiscence and return to theatre were compared between standard dressings and NPWT using logistic regression, controlled for grade of tumour, age, BMI, smoking status, diabetes and previous surgery.

Results There were 352 patients in the standard dressing group and 42 patients in the NPWT group with baseline difference in the smoking status and age. The mean age was 60 (range 25-91). The mean BMI was 37 (range 15-74). NPWT did not decrease the SSI rate $(p=0.641)$ and return to theatre (0.226), but decreased the wound dehiscence rate $(p=0.021$, OR 2.773). Higher BMI was found to increase the SSI rate $(\mathrm{p}=0.001$, OR 1.415).

Conclusions The results of this study suggest that negative pressure wound therapy does not decrease SSI rate, but decreases the wound dehiscence rate. Further randomised control trials in gynaecological oncology patients undergoing laparotomy are needed, especially for higher risk groups, such as obese patients. 